\title{
Vitamin D supplementation and hemoglobin: dosing matters in prevention/ treatment of anemia
}

\author{
Lena M. Napolitano(1)
}

\section{To the Editor:}

Arabi et al. report in the recent systematic review and meta-analysis of the effect of vitamin D supplementation on hemoglobin concentration that supplementation with vitamin $\mathrm{D}$ had no significant effect on hemoglobin and ferritin levels, while positive effects on transferrin saturation and iron status were observed [1]. There is concern that the studies in this meta-analysis included highly variable patient cohorts and vitamin D dosing, acute and chronic treatment strategies, and not all patients had anemia.

The potential beneficial impact of vitamin $D$ on hemoglobin in patients with anemia was not separately examined. Vitamin D supplementation may provide a safe, simple and cost-effective therapy for prevention and/or treatment of anemia. There is concern that the conclusion of this meta-analysis “... vitamin D had no significant effect on hemoglobin levels" is not meaningful based on the wide diversity of studies included. Why would vitamin D impact on hemoglobin if hemoglobin levels are normal?
Did the authors consider assessment of the total vitamin $\mathrm{D}$ dose in each of the studies, and separate analysis of the trials in anemic patients? The Table below confirms the wide variability in total dose and duration in these randomized clinical trials, which makes it challenging to discern an impact of vitamin $\mathrm{D}$ on hemoglobin in anemic patients.

It has clearly been documented that high-dose vitamin D is required for hepcidin suppression, which is required for optimal increase in endogenous erythropoiesis and subsequent increase in hemoglobin in anemic patients who have high hepcidin levels and anemia of inflammation or chronic kidney disease [2-7]. We would like to highlight the very important finding in Fig. 2 (effect of vitamin D on hemoglobin) in 2.3.6 "Critically ill patients", that the placebo cohort mean hemoglobin was $6.7 \mathrm{~g} / \mathrm{dL}$ vs. $11.25 \mathrm{~g} / \mathrm{dL}$ in the high-dose $(500,000$ units total, 100,000 units daily for 5 days) vitamin $\mathrm{D}$ intervention cohort.

It should also be noted that in Table 1 for the Smith study in mechanically ventilated critically ill adults, the duration of the vitamin $\mathrm{D}$ intervention was listed as "4 weeks", but the study drug was administered in 5 equal doses over 5 days, not 4 weeks. There are additional errors in that Table regarding drug dosing, duration and year of publication, all corrected in the Table below. 
Table 1 Wide variability in Vitamin D dose and duration, type of patients enrolled, and whether anemia was present or not in randomized clinical trials

\begin{tabular}{|c|c|c|c|c|c|}
\hline & Patients & Anemia & Vitamin D dose & $\begin{array}{l}\text { Vitamin D } \\
\text { duration }\end{array}$ & $\begin{array}{l}\text { Total Vitamin D } \\
\text { dose }\end{array}$ \\
\hline Trautveffer 2014 & Healthy adults & No & Fortified bread with 400 IU vit D3 daily & 8 weeks & $22,400 \mathrm{IU}$ vit D3 \\
\hline Miskulin 2016 & Adults with CKD & Yes & Oral dose of 50,000 IU vit D2 weekly & 6 months & 1,200,000 IU vit D2 \\
\hline Toxqui 2013 & Adult Women & Yes & $\begin{array}{l}\text { Fortified milk with } 200 \text { IU vit D3 \& } 15 \text { mg } \\
\text { iron daily }\end{array}$ & 16 weeks & $22,400 \mathrm{IU}$ vit D3 \\
\hline Sooragonda 2015 & Healthy adults & Yes & 0.6 Lakh Amp vit D3+ Amp iron once & Once & 600,000 IU vit D3 \\
\hline Smith $2017[7]^{a}$ & Healthy adults & No & Oral dose of $250,000 \mathrm{IU}$, vit D3 once & Once & 250,000 IU vit D3 \\
\hline Smith 2018 [6] & Critically ill adults & Yes & $\begin{array}{l}\text { Enteral dose of } 50,000 \text { IU (I1) or 100,000 IU } \\
\text { (I2) vit D3 daily }\end{array}$ & 5 days & $\begin{array}{l}500,000 \mathrm{IU}(\mathrm{I} 1) \text { vit D3 } \\
250,000 \mathrm{IU}(\mathrm{I} 2) \text { vit D3 }\end{array}$ \\
\hline Madar 2016 & Healthy adults & No & $\begin{array}{l}\text { I1 = tablet } 1000 \mathrm{IU}, \text { vit D3 daily } \\
\mathrm{I} 2 \text { = tablet } 400 \mathrm{IU}, \text { vit D3 daily }\end{array}$ & 16 weeks & $\begin{array}{l}42,000 \mathrm{IU}(\mathrm{I} 1) \text { vit D3 } \\
16,800 \mathrm{IU}(\mathrm{I}) \text { vit D3 }\end{array}$ \\
\hline Hennigar 2016 & Healthy adults & No & $\begin{array}{l}\text { Fortified bar with } 1000 \text { IU vit D3 + } \\
2000 \text { mg calcium, } 2 \text { bars per day }\end{array}$ & 9 weeks & $126,000 \mathrm{IU}$ vit D3 \\
\hline Ernest 2016 & $\begin{array}{l}\text { Adults with } \\
\text { hypertension }\end{array}$ & No & Oral dose of 2800 IU vit D3 daily & 8 weeks & 156,800 IU vit D3 \\
\hline Ernest 2017 & Adults with heart failure & $\begin{array}{l}\text { Some }(23 \\
\text { of } 172)\end{array}$ & Oral dose of $4000 \mathrm{IU}$ vit D3 daily & 36 months & 4,032,000 IU vit D3 \\
\hline Jastrzebska 2017 & Healthy adults & No & Oral dose of 5000 IU vit D3 daily & 8 weeks & $280,000 \mathrm{IU}$ vit D3 \\
\hline Dahlquist 2017 & Healthy adults & No & Sport drink with $5000 \mathrm{IU}$ vit D3 once & Once & 5000 IU vit D3 \\
\hline Walentukiewicz $2018^{a}$ & Elderly women & No & Oral dose of 28,000 IU vit D3 weekly & 12 weeks & 336,000 IU vit D3 \\
\hline Panwar 2018 & Adults with CKD & Yes & $\begin{array}{l}\text { Oral dose of } 0.5 \text { mcg (20 IU vit D3) } \\
\text { calcitriol daily }\end{array}$ & 6 weeks & $840 \mathrm{IU}$ vit D3 \\
\hline
\end{tabular}

CKD Chronic kidney disease

${ }^{\mathrm{a}}$ Hemoglobin not reported

\section{Acknowledgements}

Not applicable.

\section{Author's contributions}

LMN conceived and wrote the paper and approved the final manuscript.

\section{Funding}

None.

Availability of data and materials

Not applicable.

\section{Declarations}

Ethics approval and consent to participate

Not applicable.

\section{Consent for publication}

Not applicable.

\section{Competing interests}

None.

Received: 1 July 2020 Accepted: 25 February 2021

Published online: 19 March 2021

\section{References}

1. Arabi MA, Ranjbar G, Bahrami LS, Vafa M, Norouzy A. The effect of vitamin D supplementation on hemoglobin concentration: a systematic review and meta-analylsis. Nutr J. 2020;19:11. https://doi.org/10.1186/s12937-020-0526-3.

2. Ganz T. Anemia of inflammation. N Engl J Med. 2019;381:1148-57.
3. Bacchetta J, Zaritsky JJ, Sea JL, Chun RF, Lisse TS, Zavala K, Nayak A, Wesseling-Perry K, Westerman M, Hollis BW, Salusky IB, Hewison M. Suppression of iron-regulatory hepcidin by vitamin D. J Am Soc Nephrol. 2014;25(3):564-72. https://doi.org/10.1681/ASN.2013040355.

4. Cherry-Bukowiec JR, Engoren M, Wiktor A, Raghavendran K, Napolitano LM. Hepcidin and Anemia in surgical critical care: a prospective cohort study. Crit Care Med. 2018;46(6):e567-74. https://doi.org/10.1097/CCM.0000000000003089.

5. Sihler KC, Raghavendran K, Westerman M, Ye W, Napolitano LM. Hepcidin in trauma: linking injury, inflammation, and anemia. J Trauma. 2010;69(4):8317. https://doi.org/10.1097/TA.0b013e3181f066d5 PMID: 20938270.

6. Smith EM, Jones JL, Han JE, Alvarez JA, Sloan JH, Konrad RJ, Zughaier SM, Martin GS, Ziegler TR, Tangpricha V. High-dose vitamin D3 administration is associated with increases in hemoglobin concentrations in mechanically ventilated critically ill adults: a pilot double-blind, randomized, placebocontrolled trial. J Parenter Enter Nutr. 2018;42(1):87-94. https://doi.org/10.11 77/0148607116678197 PMID: 29505145.

7. Smith EM, Alvarez JA, Kearns MD, Hao L, Sloan JH, Konrad RJ, Ziegler TR, Zughaier SM, Tangpricha V. high-dose vitamin D3 reduces circulating hepcidin concentrations: a pilot, randomized, double-blind, placebocontrolled trial in healthy adults. Clin Nutr. 2017;36(4):980-5. https://doi. org/10.1016/j.clnu.2016.06.015 PMID: 27402475.

\section{Publisher's Note}

Springer Nature remains neutral with regard to jurisdictional claims in published maps and institutional affiliations. 